\title{
Menorrhagia, CTCAE
}

National Cancer Institute

\section{Source}

National Cancer Institute. Menorrhagia, CT CAE. NCI Thesaurus. Code C143671.

A disorder characterized by abnormally heavy vaginal bleeding during menses. 